\title{
Advanced Methodology and Measurements of Material and Mechanical Properties of Heart Valves Under Dynamic Strain
}

\author{
R Madhurapantula ${ }^{1}$, J Orgel ${ }^{1}$ \\ ${ }^{1}$ Illinois Institute of Technology, Chicago, IL \\ rmadhura@iit.edu
}

Mammalian heart valves are complex soft tissue assemblies with diffuse boundaries between constituent tissue types, i.e. leaflet (LL), chordae tendinae (CT) and papillary muscles (PM). In mitral and tricuspid valves, the LL are connected to the CT which connect to the ventricular wall on the other end by interfacing with PM. Each of these constituent tissue elements are markedly different in molecular packing and architecture, and hence mechanical properties from these tissue boundaries or interfaces. The material composition and the mechanical properties, as reported here, were not previously available for these tissue assemblies. We present here, developments in multi-scale methodologies using X-diffraction mapping and microscopic evaluation of the application of dynamic strain on these assemblies. The correlation between changes in "molecular strain" in comparison to macro and microscopic strain provide insights in to possible points of failure in these tissues. This information is of great value in developing novel heart valve implants, improving the surgical simulators and aide in developing new methods of teaching surgical techniques, and assisting with robot-assisted surgery.
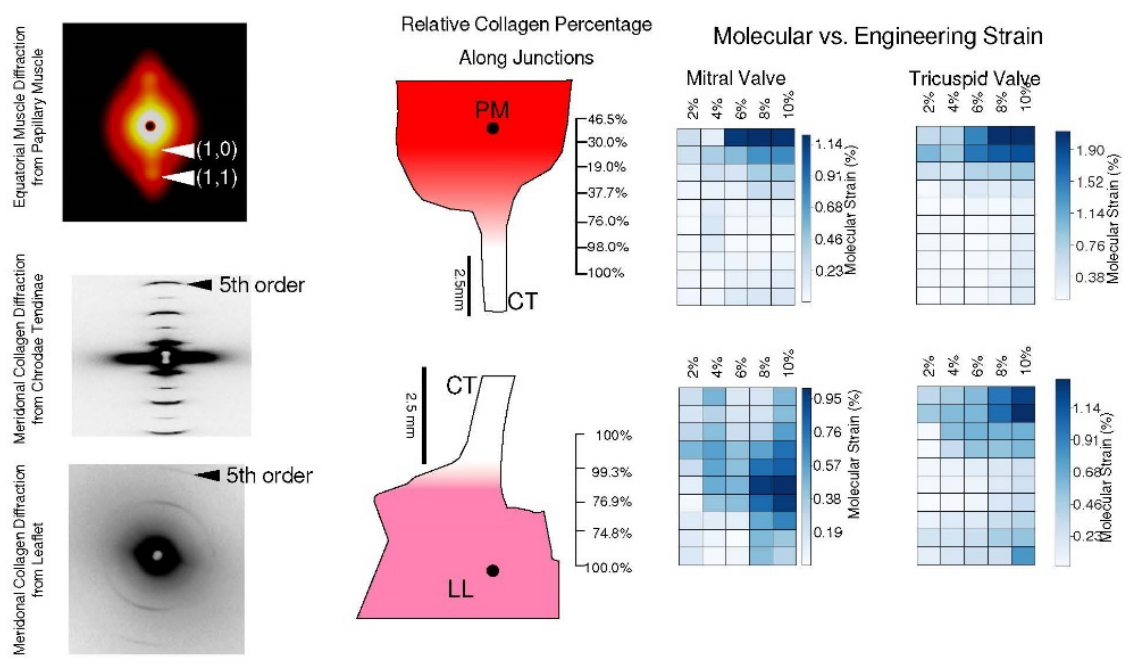

Points of Mechanical Failure in Valves
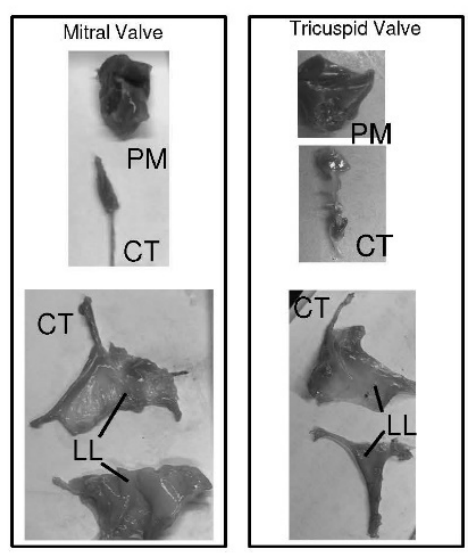

Figure 1 